Revue pluridisciplinaire d'études médiévales

42 | 2021

Fama : réputation et renommée

\title{
Développer et faire perdurer sa renommée au Moyen Âge. Le cas de Merlin dans les Suites du Merlin en prose ( $\mathrm{XIII}^{\mathrm{e}}$ siècle)
}

\section{Lise Fuertes}

\section{OpenEdition}

Édition électronique

URL : https://journals.openedition.org/questes/5682

DOI : 10.4000/questes.5682

ISSN : 2109-9472

Éditeur

Les Amis de Questes

\section{Édition imprimée}

Date de publication : 28 janvier 2021

Pagination : 53-65

ISSN : 2102-7188

\section{Référence électronique}

Lise Fuertes, « Développer et faire perdurer sa renommée au Moyen Âge. Le cas de Merlin dans les Suites du Merlin en prose (xIII siècle) », Questes [En ligne], 42 | 2021, mis en ligne le 28 février 2021, consulté le 06 février 2022. URL : http://journals.openedition.org/questes/5682 ; DOI : https://doi.org/ 10.4000 /questes.5682 


\title{
Développer et faire perdurer sa renommée au Moyen Âge. Le cas de Merlin dans les Suites du Merlin en prose ( $\mathrm{XIII}^{\mathrm{e}}$ siècle)
}

\author{
Lise FUERTES \\ Université de Bourgogne - Laboratoire CPTC
}

Au début du $\mathrm{XIII}^{\mathrm{e}}$ siècle, le Merlin $^{1}$ en prose, premier roman à mettre en scène Merlin en tant que personnage éponyme, construit la « bonne renommée ${ }^{2}$ » de celui-ci, fondée sur des qualités de sage et de prophète. Il devient le conseiller respecté d'Uterpandragon, le père d'Arthur, puis aide ce dernier à accéder au trône.

Toutefois, dans le roman arthurien, Merlin est la plupart du temps mentionné uniquement dans des analepses ${ }^{3}$. C'est le cas au début du Lancelot en prose $^{4}$, souvent intégré au cycle Lancelot-Graal ${ }^{5}$. Merlin disparu reste renommé

\footnotetext{
${ }^{1}$ Robert de Boron, Merlin, roman du XIII siècle, éd. Alexandre Micha, Genève, Droz, 2000.

${ }^{2}$ Lorsque nous parlerons de la renommée de Merlin dans le sens de « reconnaissance de ses qualités », nous emploierons désormais l'expression de « bonne renommée ».

${ }^{3}$ Paul Zumthor, «La délivrance de Merlin : contribution à l'étude des romans de la Table Ronde », Zeitschrift für romanische Philologie, vol. 62, 1942, p. 370-386, cit. p. 371.

${ }^{4}$ Nous nous référerons à plusieurs éditions critiques de ce texte : Lancelot. Roman en prose $d u$ XIII siècle, éd. Alexandre Micha, Paris/Genève, Droz, 1978-1982, 8 vol. ; Lancelot du Lac, roman français du XIII siècle, éd. François Moses, Paris, Le Livre de Poche, coll. « Lettres Gothiques », 1991 ; Lancelot, dans Le Livre du Graal, éd. Philippe Walter, Paris, Gallimard, coll. « Bibliothèque de la Pléiade », 2003-2009, 2 vol.

${ }^{5}$ Un cycle se définit comme un assemblage de branches autonomes, dotées d'une « unité thématique et d'une successivité chronologique », mais soumises à une "totalité qui les subsume » (Annie Combes, Les voies de l'aventure. Réécriture et composition romanesque dans le Lancelot en prose, Paris, Honoré Champion, 2001, p. 55, et Nathalie Koble, « Les romans arthuriens en prose au XIII ${ }^{\mathrm{e}}$ siècle : des cycles en série ? ", dans Cycle et collection, dir. Anne Besson, Vincent Ferré et Christophe Pradeau, Paris, L'Harmattan, 2008, p. 179-198, cit. p. 18).
} 
pour la véracité de ses prophéties ${ }^{6}$, ce qui lui accorde la faveur du petit peuple ${ }^{7}$, donc une certaine popularité. À l'inverse, la voix publique rappelle constamment sa réputation de fils de diable, quoique parfois compensée par les mentions de son baptême et de sa rédemption ${ }^{8}$. Malgré des variations plus positives ${ }^{9}$, sa disparition est également présentée comme la conséquence de cette mauvaise réputation.

Néanmoins, à partir du milieu du XIII ${ }^{\mathrm{e}}$ siècle, sont rédigées trois Suites du Merlin rétrospectives ${ }^{10}$ et elleptiques ${ }^{11}$. La Suite dite « Vulgate » est la version la plus fréquente, à laquelle nous nous référerons ici dans sa version courte intitulée Les Premiers Faits du roi Arthur ${ }^{12}$. Probablement composée en opposition à la première $^{13}$, la Suite dite « post-Vulgate ${ }^{14} »$ ne semble pas appartenir à un cycle ${ }^{15}$. Enfin, le Livre d' 'Artus ${ }^{16}$, ainsi intitulé par H. Oskar Sommer, est considéré comme la « suite » qui poursuit la Suite Vulgate version longue dans le seul manuscrit où elle subsiste, le Bnf fr. 337. Quelles que soient leurs particularités codicologiques,

${ }^{6}$ Lancelot, éd. Walter, vol. 2, « Galehaut», § 45, p. 974 et $\S 46$, p. 975.

${ }^{7}$ Lancelot, éd. Moses, p. 90 et éd. Micha, vol. 7, chap. VIa, p. 38-41.

${ }^{8}$ C'est le cas dans l'édition Pléiade du texte, qui se fonde sur le manuscrit Universitätbibliothek Bonn S 526 contenant l'ensemble du cycle Lancelot-Graal : Lancelot, éd. Walter, vol. 2, « La Marche de Gaule », § 43, p. 44.

${ }^{9}$ Ibid., § 44, p. 47.

${ }^{10}$ Elles sont composées après les romans qu'elles prétendent précéder : le Lancelot, la Quête du Saint Graal et la Mort du roi Arthur.

${ }^{11}$ Une suite elleptique a pour fonction de «combler une lacune ou une ellipse médiane » (Gérard Genette, Palimpsestes. La littérature au second degré, Paris, Éditions du Seuil, 1982, p. 242), ici entre les premières années du règne d'Arthur relatées dans le Merlin et sa souveraineté dans la plénitude de l'âge, dans le Lancelot.

${ }^{12}$ Les Premiers Faits du roi Arthur, éd. Anne Berthelot et Philippe Walter, dans Le Livre du Graal, Paris, Gallimard, Bibliothèque de la Pléiade, vol. 1, 2001, p. 809-1662. Sur la tradition manuscrite de ce texte, voir Richard Trachsler, " Pour une nouvelle édition de la Suite Vulgate du Merlin », Vox Romanica, n 60, 2001, p. 128-148, et Patrick Moran, Lectures cycliques. Le réseau inter-romanesque dans les cycles du Graal du XIII siècle, Paris, Honoré Champion, 2014, p. 501-542.

${ }^{13}$ Nathalie Koble, « Les romans arthuriens en prose », art. cit., p. 184.

${ }^{14}$ Nous utiliserons l'édition suivante : La Suite du Roman de Merlin, éd. Gilles Roussineau, Genève, Droz, 2006.

${ }^{15}$ La Suite du Roman de Merlin, éd. cit., p. XXXIII.

${ }^{16}$ Le Livre d'Artus, dans The Vulgate Version of the Arthurian Romances, éd. H. Oskar Sommer, Washington, Carnegie Institution, 1913, t. 7, Supplément. Voir Patrick Moran, Lectures cycliques..., op. cit., p. 501-542. 
littéraires ou cycliques, ces romans remettent Merlin au centre du récit puis le font disparaître.

Par conséquent, comment le personnage utilise-t-il sa parole prophétique dans cet espace textuel, à la fois pour développer sa bonne renommée et pour éviter que sa disparition inéluctable - mort ou enfermement définitif - ne le condamne à l'oubli ${ }^{17}$ ? À la fin des Suites, lorsqu'il a disparu, sur quelle tonalité son souvenir survit-il ? Loin de ne concerner que la caractérisation du personnage, les variations de sa renommée s'intègrent à des enjeux poétiques essentiels.

Pour chaque Suite, nous confronterons les interventions prophétiques de Merlin avant sa disparition aux modalités de la survivance de sa bonne renommée, auprès des autres personnages ${ }^{18}$ et du lecteur.

\section{Les Premiers Faits du roi Arthur: de la bonne renommée à la légende}

Dans Les Premiers Faits du roi Arthur, Merlin hérite sans conteste de l'honorabilité qui le caractérise à la fin du Merlin, grâce à des qualités morales qui lui valent le nom de « prodome ». Pourtant, l'imminence de sa disparition le pousse à se construire une légende, au sens étymologique du terme, c'est-à-dire à influencer « ce qui sera lu » ou ce qui sera dit de lui après son départ. Dans le sillage du Merlin, qui fait de lui l'auteur fictif du récit, Merlin devient ici autobiographe pour renforcer sa bonne renommée.

Il profère ainsi ce que nous qualifions d' « auto-prophéties » : des segments prophétiques dans lesquels il évoque sa propre destinée. Très tôt dans le texte, il

\footnotetext{
${ }^{17}$ Le Moyen Âge associe la mort et la renommée, pour souligner que la pérennité de la seconde est le seul moyen de vaincre la première (Jacqueline Cerquiglini-Toulet, « Fama et les preux : nom et renom à la fin du Moyen Âge », Médiévales, n 24, 1993, p. 35-44), que l'immortalité céleste dépend de la renommée terrestre (Philippe Ariès, Essai sur l'histoire de la mort en Occident du Moyen Âge à nos jours, Paris, Seuil, 1975, p. 88) ou, au contraire, que la renommée terrestre demeure vaine (Johan Huizinga, L'automne du Moyen Âge [1932], trad. Julia Bastin, Paris, Payot, 2002, p. 212-214).

${ }^{18}$ Claude Gauvard affirme en effet que les personnages non-nobles « n'existent que par les yeux des autres » («La fama, une parole fondatrice », Médiévales, n² 24, 1993, La Renommée, p. 513, cit. p. 11).
} 
se dépeint ainsi sous les traits d'un «lyon sauvage » qu'une « leuve » viendra « loiier de cerceles ${ }^{19} »$. Le masque allégorique du lion sauvage, qualité valorisée dans Les Premiers Faits du roi Arthur, lui permet de s'affirmer comme l'égal du roi Arthur, désigné par le même signifiant animal dans les prophéties de son conseiller.

Bien plus, la «leuve» Niniane transforme sa disparition en destin pathétique légendaire. Devenue l'amie de Merlin et son élève en magie, celle-ci met en pratique son savoir occulte pour « l'enserrer » dans une tour merveilleuse faite d'air, dont il ne pourra pas s'échapper ${ }^{20}$.

Néanmoins, une légende ne peut exister sans être diffusée. La dernière autoprophétie de Merlin à Blaise en témoigne :

Et Merlins li dist : «Faites moi tés letres que je vous deviserai, et lors si saurés quanques vous i porrés aïdier. » Et il les fist, si fu tels li contes : «Ce est li commencemens et li contes des aventures du païs par coi li merveillous lyons fu enserrés et que fix de roi et de roïne descendra et covenra que il soit chastes et li miudres chevaliers del monde $\gg{ }^{21}$.

Bien que ces paroles semblent faire allusion à Galaad, fils d'un roi et d'une reine et désigné comme un « lion » dans certaines prophéties du Lancelot $^{22}$, elles ne peuvent en réalité annoncer la destinée du jeune homme, puisque celui-ci n'est pas «enserrés" à la fin de sa vie. Seule la dernière partie de la proposition s'applique donc au chevalier, sans que les variations ${ }^{23}$ rencontrées dans les manuscrits de la Suite Vulgate ne modifient ce sens. Merlin renforce donc bien son propre éloge sous le masque laudatif du « merveillous lyons [...] enserrés ».

\footnotetext{
${ }^{19}$ Les Premiers Faits du roi Arthur, § 249, p. 1050. Malgré une variante (« lupart sauvage »), relevée par Richard Trachsler, la majorité des copistes de la Suite Vulgate emploie le signifiant « lion » (Clôtures du cycle arthurien, études et textes, Genève, Droz, 1996, p. 85-86).

${ }^{20}$ Les Premiers Faits du roi Arthur, éd. cit., § 810, p. 1631-1632.

${ }^{21}$ Ibid., § 652, p. 1450.

${ }^{22}$ Lancelot, éd. Walter, vol. 3, « La seconde partie de la quête de Lancelot », § 209, p. 229-230.

${ }^{23}$ Richard Trachsler, Clôtures du cycle arthurien..., op. cit., p. 89.
} 
La diffusion de cette légende est détaillée et fondée sur le double sens du terme « letres » en français médiéval. D’abord, Merlin se charge lui-même de diffuser ses prophéties et auto-prophéties recopiées par Blaise sous forme épistolaire $^{24}$, sur les chemins par lesquels passent les chevaliers. Se trouve donc représentée ici concrètement l'origine des différentes inscriptions retrouvées par les chevaliers dans le Lancelot $^{25}$. Par conséquent, Merlin se fait figure allégorique de la renommée qui « vole », pour assurer la pérennité de sa légende dans le futur diégétique ${ }^{26}$.

Surtout, la légende de Merlin entre en littérature. Le terme « letres » signifie également ici le contenu d'un livre. Grâce au mot « contes » et à l'introduction de l'auto-prophétie, le prosateur semble écrire le prologue d'un roman. Cet usage du métalangage littéraire rapproche donc cette mise en écrit de la «pronunciatio » ou dictée, façon possible de créer l'œuvre littéraire au Moyen Âge ${ }^{27}$. Par conséquent, Merlin assure aussi la propagation de sa légende en direction du lecteur médiéval ou moderne qui lirait le cycle Lancelot-Graal dans son ordre diégétique ${ }^{28}$.

Après son enserrement, Merlin ne profère plus d'auto-prophétie. Néanmoins, sa voix prophétique continue à faire vivre sa légende de " prodome » et de prophète à travers sa prison d'air. En effet, il parle une dernière fois à Gauvain $^{29}$, lors de la quête qu'Arthur a lancée pour retrouver son fidèle conseiller ${ }^{30}$. Après s'être plaint de sa destinée fatale conforme à la légende qu'il s'est forgée, Merlin révèle au chevalier, transformé en nain pour avoir omis de

\footnotetext{
${ }^{24}$ Les Premiers Faits du roi Arthur, éd. cit., $\S 652$ et $\S 653$, p. 1450.

${ }^{25}$ Lancelot, éd. Walter, vol. 2, « La Première Partie de la quête de Lancelot », § 232, p. 1670.

${ }^{26}$ Paul Zumthor, Merlin le prophète, un thème de la littérature polémique, de l'historiographie et des romans [Lausanne, Payot, 1943], Genève, Slatkine, 2000, p. 170.

${ }^{27}$ Paul Zumthor, La lettre et la voix, De la «littérature » médiévale, Paris, Éditions du Seuil, 1987, p. 111-112.

${ }^{28}$ Patrick Moran distingue plusieurs formes de réception du cycle : une lecture linéaire (dans l'ordre chronologique), tabulaire (par réseau) ou modulaire (chaque branche indépendamment des autres). Voir Lectures cycliques..., op. cit., p. 332-336.

${ }^{29}$ Les Premiers Faits du roi Arthur, éd. cit., § 827-830, p. 1651-1653.

${ }^{30}$ Ibid., § 811, p. 1633.
} 
saluer une fée, comment retrouver son apparence originelle ${ }^{31}$. Ces ultimes paroles sont diffusées, car mises par écrit au retour de Gauvain à la cour, selon la coutume établie $^{32}$.

Les conséquences poétiques et idéologiques de la transformation du destin merlinien en légende ne sont pas négligeables. La version du Lancelot poursuivant Les Premiers Faits du roi Arthur relit en effet la vie et la disparition de Merlin sous une lumière positive. Par conséquent, la légende de Merlin a d'abord valeur de «marque de cyclicité ${ }^{33} »$, c'est-à-dire d'élément établissant un lien structurel fort entre deux branches différentes du cycle Lancelot-Graal.

De manière plus subtile, la légende de Merlin valide son autorité d'auteur. En effet, en tant qu'histoire du Graal depuis sa perte jusqu'à sa réapparition, le cycle Lancelot-Graal mime le récit biblique. Par son statut d'auteur fictif, Merlin se substitue alors à Dieu, seul créateur autorisé au Moyen Âge. Toutefois, sa parole reste sujette à caution du fait de ses origines diaboliques ${ }^{34}$. Se dessine alors la seconde raison de ce travail effréné de construction et de diffusion de sa légende par le personnage : en tant que conteur des aventures du saint Graal, Merlin se doit de diffuser une parole irréprochable, fondée sur une réputation impeccable.

\section{La Suite du Roman de Merlin : entre mort infamante et rétablissement}

\section{de la bonne renommée}

Dans la Suite du Roman de Merlin, la réputation de Merlin fils du diable

\footnotetext{
${ }^{31}$ Ibid., § 829, p. 1653.

${ }^{32}$ Ibid., § 524, p. 1319-1320.

${ }^{33}$ Nathalie Koble, « Les romans arthuriens en prose », art. cit., p. 190.

${ }^{34}$ Sur les problèmes d'autorité du récit liés à la figure de Merlin comme auteur, voir Alexandre Leupin, « Qui parle ? Narrateurs et scripteurs dans la Vulgate arthurienne », Digraphe, vol. 20, 1979, p. 83-109; Mireille Séguy, « Le point aveugle. La fabrique du récit fictionnel dans le Merlin en prose de Robert de Boron ", Revue de littérature française et comparée, $\mathrm{n}^{\circ} 15,2000$, p. 27-35, et Christine Ferlampin-Acher, « La parole dans le Merlin de Robert de Boron », dans Merlin, roman du XIII siècle, Robert de Boron, dir. Danielle Quéruel et Christine FerlampinAcher, Paris, Ellipses, 2000, p. 89-104.
} 
resurgit. Néanmoins, il bénéficie d'une sorte de « réhabilitation », aux yeux du lecteur comme des personnages.

À l'inverse de ce que l'on constate dans Les Premiers Faits du roi Arthur, Merlin concentre ses auto-prophéties sur l'annonce de sa mort, effectuée en termes relativement neutres et factuels ${ }^{35}$. La première, adressée à Arthur, attire pourtant l'attention sur le thème de l'honorabilité ternie :

«Et poés bien dire que nos mors sont moult divierses, la moie et la toie. - Por coi, fait li rois, iche me dites ? - Pour chou, fait il, que tu morras a hounour et jou a honte. Et seras richement ensevelis et je serai tous vis mis en terre, et c'est bien honteuse mort. » Li rois se saingne de la parole qu'il entent, si dist : «Coument ! Merlins, si morras si deshonnereement comme vous me dites ? - Voire, fait Merlins, ne je ne voi chose qui destorner m'en puisse, fors Diex seulement - Che est merveille, fait li rois, quant par ton grant sens ne te pues destorner de si grant mesaventure comme tu contes a moi. $\gg{ }^{36}$.

Les termes « honte ${ }^{37} »$ et « deshonnereement ${ }^{38} »$ évoquent l'infamie ou la privation de la bona fama $^{39}$, conséquences de l'ensevelissement «tous vis » de Merlin. Puni de sa concupiscence pour Nivienne, avatar de la déesse vierge Diane $^{40}$, Merlin sera en effet jeté dans une tombe où gisent déjà, comble de l'ironie, deux amants fidèles ${ }^{41}$. Il parait donc à juste titre « honni » par Nivienne,

\footnotetext{
${ }^{35}$ La Suite du Roman de Merlin, éd. cit., § 154, p. 118, § 281, p. 238 et § 340, p. 296.

${ }^{36}$ Ibid., § 42, p. 31-32.

${ }^{37}$ Olivier Bertrand et Silvère Menegaldo soulignent que le terme « honte » possède un sens plus fort en français médiéval qu'en français moderne (Vocabulaire d'ancien français, Paris, Armand Colin, 2006, p. 151).

38 Stéphane Marcotte traduit très justement ce terme par « infamante » (La Suite du Roman de Merlin, trad. Stéphane Marcotte, Paris, Honoré Champion, 2006, § 42, p. 150).

${ }^{39}$ Le mot infâme est emprunté au latin infamis « décrié, perdu de réputation », dérivé par préfixation de in- et de fama (Dictionnaire historique de la langue française [1972], dir. Alain Rey, Paris, Le Robert, 2012, 3 vol., article « infâme », vol. 2, p. 1720).

${ }^{40}$ Laurence Harf-Lancner, Les Fées au Moyen Âge. Morgane et Mélusine, Genève, Slatkine, 1984, p. 233.

${ }^{41}$ La Suite du Roman de Merlin, éd. cit., § 385-386, p. 334-335.
} 
c'est-à-dire «mis à mort de manière humiliante ${ }^{42}$ ». De surcroît, cette façon de mourir « hors catégorie » fait de lui un paria. Enfin, la prescience du prophète, l'un des traits sur lesquels s'appuie précisément sa bonne renommée, n'empêche pas sa mort ignominieuse : c'est le sens de la remarque et du geste d'étonnement d'Arthur.

La proclamation par Merlin de son caractère mal famé peut toutefois surprendre. En réalité, ce discours prophétique dissimule un avertissement destiné à Arthur. Il s'insère dans une série de prophéties ${ }^{43}$, de songes ou d'apparitions fantastiques ${ }^{44}$ qui annoncent à mots couverts la mort du souverain sous les coups de son fils incestueux. La honte d'Arthur porterait alors sur le péché d'inceste dont il s'est rendu coupable, détaillé dès le début de La Suite du Roman de $\operatorname{Merlin}^{45}$. Ainsi, Merlin ne ferait allusion à sa propre infamie que pour mieux rappeler celle, plus grave, du roi ${ }^{46}$. Par conséquent, la mauvaise réputation autoproclamée de Merlin reste d'une portée limitée. En outre, les prophéties merliniennes ne sont pas encore diffusées par écrit à la cour à ce moment de l'histoire ${ }^{47}$.

En revanche, Merlin s'emploie malgré lui à ternir sa réputation auprès du lecteur. Il crée divers monuments magiques qu'il souhaite pérennes ${ }^{48}$. Cependant, en tant que manifestations de sa « nigromanchie» issue des forces obscures, ses

\footnotetext{
${ }^{42}$ Laurence Hélix, L'Épreuve de vocabulaire d'ancien français, fiches de sémantique, Paris, Éditions du Temps, 1999, p. 137.

${ }^{43}$ La Suite du Roman de Merlin, éd. cit., § 11, p. 8 ; § 15, p. 12 ; § 16, p. 13, § 73, p. 56.

${ }^{44}$ Juste après l'inceste avec sa sœur la reine d'Orcanie, Arthur voit en songe un grand dragon détruire son royaume et le blesser à mort (ibid., $\S 3$, p. 2). Il rencontre ensuite la diverse beste portant en elle des chiens vivants qui aboient (ibid., §5-7, p. 3-5), interprétée comme une prophétie par l'image du châtiment de son inceste (Francis Dubost, L'Autre, l'Ailleurs, l'Autrefois. Aspects fantastiques de la littérature médiévale [XII - -XIII ${ }^{e}$ siècles], Paris, Honoré Champion, 1991, 2 vol., chap. 17, p. 511).

${ }^{45}$ La Suite du Roman de Merlin, éd. cit., § 1-2, p. 1.

${ }^{46}$ Nous remercions Lisa Sancho pour cette suggestion de lecture.

${ }^{47}$ Cette demande de mise en écrit interviendra plus tard (ibid., § 281, p. 238). Elle est faite par Merlin lui-même, qui toutefois ne délivre plus d'auto-prophétie ensuite.

${ }^{48}$ Par exemple, Merlin fait brûler des mauvais enchanteurs dans un feu qui ne s'éteindra que le jour de la mort d'Arthur (ibid., § 340, p. 296).
} 
actions ne font qu'entériner sa réputation diabolique. Cet échec trahit enfin son orgueil, la pire des raisons de sa recherche d'une bonne renommée dans les Suites.

Toutefois, après sa mort, Merlin semble réussir à faire perdurer sa bonne renommée à la cour, voire à se réhabiliter aux yeux du lecteur.

Par le biais de Baudemagu, qui a parlé à Merlin juste avant que celui-ci ne meure dans sa tombe, le roi Arthur apprend le destin de son ami, sans savoir qui en est responsable :

Et quant le roy a tout escouté, il respont: «Par mon chief, il en fait bien a croire. Il [Baudemagu] ne nous le mandast mie se il ne le sceust vraiement. Ha! Dieux, or puet on bien dire le royaume de Logres, quant Merlins le sage est mors, qu'il est moult abaissiés, car tant comme il vesquist il ne feist s'amender non et en pouvoir et en honneur. [...] » Moult est le roy doulens et corrouciés de la mort Merlin, car ilz avoient si grand fiance en luy qu'ilz ne cuidoient pas que le royaume de Logres peust jamés avoir deshonneur tant comme Merlin vesquist ${ }^{49}$.

Rappel inversé de l'auto-prophétie de Merlin sur sa propre honte, cet éloge confirme le caractère de « prodome » de Merlin. Les paroles du roi le font même accéder au statut de « sauveur » du royaume de Logres, s'il avait vécu. Après cet hommage appuyé, plus aucune rumeur ne peut entamer sa respectabilité. Ainsi, son silence habile sur la raison de son entombement lors de son dialogue avec Baudemagu porte ses fruits.

Surtout, la réhabilitation de Merlin aux yeux du lecteur est amorcée lorsque son ultime prophétie se réalise. Merlin avait annoncé à Baudemagu que Gauvain et le Morholt, prisonniers de la Roche aux Pucelles, ne pourraient être délivrés que par Gaheriet, encore écuyer ${ }^{50}$. Adoubé par Arthur ${ }^{51}$, Gaheriet délivre effectivement ses compagnons ${ }^{52}$. La performativité de la parole prophétique

\footnotetext{
${ }^{49}$ Ibid., § 527-528, p. 499-500.

${ }^{50}$ Ibid., § 526, p. 497-498.

${ }^{51}$ Ibid., $\S 532$, p. 504.

${ }^{52}$ Ibid., § 574-578, p. 557-559.
} 
merlinienne efface alors la mauvaise réputation du fils du diable, qui retrouve sa bonne renommée de prophète influençant positivement l'action.

Finalement, ce mouvement contradictoire de flétrissure infligée à la réputation de Merlin avant sa mort et de réaffirmation post-mortem de sa bonne renommée possède l'avantage de faire correspondre la Suite à toutes les versions du Lancelot, même celles présentant la réputation de Merlin sous un jour défavorable. Se justifie alors la conception moderne de la Suite Post-Vulgate comme une « suite cyclique sans cycle $^{53} »$ manifestant un fort tropisme vers le cycle Lancelot-Graal.

Enfin, dans un récit centré sur les merveilles du Graal, le choix de réaffirmer in fine la bonne renommée de Merlin renforce encore une fois véracité de sa parole.

\section{Le Livre d'Artus : le silence sur la renommée?}

Le prosateur du Livre d'Artus choisit une solution plus radicale : ne pas mentionner la bonne renommée de Merlin avant sa disparition. Lorsque le prophète a disparu, le motif de ses qualités proverbiales resurgit toutefois, avec une tonalité plus ambigüe.

Il paraît en effet difficile de parler « d'auto-prophétie » à propos du segment prophétique suivant :

Et [Merlin] dist que voirement estoit ele [Niniane] femme du lignage au lou car quant plus grant bien li fait ses maistres et il pis li fait ja tant ne l'avra norri petit feon. Et tex est costume de femme que ja tant ne li savra en bien faire en quel que maniere que ce soit que ele ne traisse celui qui toz les biens li aura fait et porchaciez a son vivant ${ }^{54}$.

\footnotetext{
${ }^{53}$ Patrick Moran, Lectures cycliques..., op. cit., p. 103.

${ }^{54}$ Livre d'Artus, éd. cit., p. 164, 1. 4-8 ; Paris, BnF fr. 337, fol. $202 \mathrm{v}$.
} 
Merlin y fait silence sur son imminente disparition. À la place, il reproche à Niniane une trahison qui a eu lieu par le passé ${ }^{55}$. De surcroît, ses paroles insistent davantage sur la réputation de la femme traîtresse, comme en témoignent la périphrase qui la compare au loup et la sentence misogyne ${ }^{56}$. Bien que Merlin rappelle discrètement ses propres qualités de bonté et de savoir, sa renommée est éclipsée par la réputation de Niniane, la future Dame du Lac.

Toutefois, on peut choisir de lire la sentence finale comme une annonce de la disparition de Merlin à cause de Niniane. Néanmoins, même alors, le personnage échoue à se construire une légende semblable à celle que l'on lit dans Les Premiers Faits du roi Arthur. À cause de l'absence du bestiaire valorisant et en tant que victime quelque peu ridicule de la duplicité bien connue des femmes, le prophète rejoint au contraire la cohorte anonyme des hommes trahis par elles.

Cette discrétion sur la renommée de Merlin nous semble coïncider avec l'incertitude qui plane sur son destin. Le prosateur du Livre d'Artus fait en effet allusion à un possible «martire » du personnage, trahi par Niniane et livré aux diables qui l'on conçu ${ }^{57}$, ce qui rappellerait sa mort dans la Suite du Roman de Merlin. Est avancée ensuite une délivrance finale du prophète par Perceval ${ }^{58}$. Cette dernière hypothèse supposerait que Merlin ait été emprisonné, peut-être dans un lieu appelé «l'esplumeoir Merlin », allusion évidente au Perceval en prose attribué à Robert de Boron ${ }^{59}$. Finalement, la dernière mention de Merlin dans la diégèse offre une version assez semblable à celle de son enserrement dans

\footnotetext{
55 Ibid., p. 126, 1. 45-48 ; Paris, BnF fr. 337, fol. 182v.

${ }^{56}$ Marie-Louise Ollier, «Proverbe et Sentence. Le discours d'autorité chez Chrétien de Troyes », Revue des Sciences Humaines, Rhétorique du proverbe, t. 41, n 163, 1976, p. 336. L'auteure distingue le proverbe de la sentence sur le critère de la brièveté. Lorsque le proverbe est développé, il devient une sentence.

${ }^{57}$ Livre d'Artus, éd. cit., p. 127, 1. 11-20 ; Paris, BnF fr. 337, fol. 183r.

${ }^{58}$ Ibid. Cette version se retrouve, selon Paul Zumthor, dans un seul manuscrit du Lancelot en prose (Paris, BnF fr. 754). Le critique postule l'existence d'un modèle perdu qui aurait mentionné à la fois l'aventure sentimentale de Merlin avec Niniane et sa délivrance (Paul Zumthor, «La délivrance de Merlin », art. cit., p. 371 et p. 383-384).

${ }^{59}$ Robert de Boron, Le roman du Graal : manuscrit de Modène, éd. Bernard Cerquiglini, Paris, Union Générale d'Édition, 1981.
} 
Les Premiers Faits du roi Arthur : il « sejornoit avec s'amie ${ }^{60} »$. Cette dernière sortie de scène imprécise ouvre néanmoins le récit sur les autres versions, puisque Niniane n'aime pas vraiment Merlin.

Par conséquent, dans un texte qui choisit délibérément de ne pas présenter une version particulière de la fin de Merlin $^{61}$, mais de s'appuyer sur l'intertexte pour en suggérer plusieurs, il n'est pas étonnant que le problème de sa renommée soit escamoté.

Cette fin indéterminée engendre assez logiquement le rappel de la réputation de Merlin, qui devient tantôt bonne, tantôt mauvaise, aux yeux des personnages et du lecteur.

Comme dans les autres Suites, Gauvain proclame encore la bonne renommée de Merlin fondée sur sa prescience, pour répondre toutefois à un « $\operatorname{gab}^{62} »$ ou remarque moqueuse. À l'inverse, par la voix du prosateur ${ }^{63}$ et de personnages divers ${ }^{64}$, de fréquentes analepses insistent sur les mauvaises coutumes magiques instaurées par Merlin dans un lieu appelé désormais « l'île Merlin ». Seul Formiz d'Armes, jeune chevalier, rappelle la bonté de Merlin, qui lui a permis de vivre avec son amie sur l'île ${ }^{65}$. Bien plus, contrairement à ce qui se passe dans les autres Suites, aucune intervention de Merlin, directe ou indirecte, ne vient rétablir sa bonne renommée ou réaffirmer son renom.

En somme, ces choix soulignent deux préoccupations poétiques essentielles du Livre d'Artus. D'abord, la renommée stéréotypée et la réputation ambigüe de Merlin servent son escamotage. Elles coïncident également avec sa mauvaise réputation dans le Lancelot. L'usage intensif de l'analepse dans le Livre d'Artus

\footnotetext{
${ }^{60}$ Livre d'Artus, éd. cit., p. 164, 1. 17 ; Paris, BnF fr. 337, fol. 202v.

${ }^{61}$ Pour les conséquences poétiques de ce choix original, nous nous permettons de renvoyer à notre article : Lise Fuertes-Regnault, «La fin de Merlin dans le Livre d'Artus : la mise à mort confisquée », L'Esplumeoir, ${ }^{\circ} 13,2014$, p. 47-68.

${ }^{62}$ Livre d'Artus, éd. cit., p. 176, 1. 29-30 ; Paris, BnF fr. 337, fol. 209v.

${ }^{63}$ Ibid., p. 301-302 ; Paris, BnF fr. 337, fol. 258r.

${ }^{64}$ Ibid., p. 304, 1. 7-16 et p. 306, 1. 21-30 ; Paris, BnF fr. 337, fol. 259r. et fol. 260v.

${ }^{65}$ Ibid., p. 310-311; Paris, BnF fr. 337, fol. 263v.
} 
traduit sans doute d'ailleurs le même effort de jointure avec son aval diégétique, bien qu'aucun manuscrit ne regroupe effectivement les deux textes.

Les trois Suites du Merlin en prose traitent la thématique de la renommée de Merlin par des procédés à la fois différents et complémentaires qui redéfinissent leurs relations les unes par rapport aux autres. Fidèle à sa tonalité optimiste, Les Premiers Faits du roi Arthur est le seul texte à montrer le développement et la propagation intacte d'une véritable légende merlinienne. Bien que ternie par sa réputation de luxure, dans la Suite du Roman de Merlin, la bonne renommée du prophète est pourtant complètement réaffirmée et maîtrisée par lui après sa mort. En cela, les deux Suites se rapprochent. À rebours des particularités codicologiques qui le relient à la Suite Vulgate, le Livre d'Artus se singularise, en ne restituant de la bonne renommée de Merlin que ce qui peut être utile à un hypothétique rattachement au cycle Lancelot-Graal. Les trois Suites s'unissent néanmoins lorsqu'elles évoquent les raisons qui poussent ce personnage non noble à rechercher le développement et la pérennité d'une bonne renommée : une volonté de se définir par son renom (quelque peu égoïste, mais conforme aux mœurs médiévales) et surtout la nécessité de fonder la vérité de sa parole d'auteur du roman du Graal sur une renommée sans tache. 
